\title{
The quantum-Ehrenfest method with the inclusion of an IR pulse: Application to electron dynamics of the allene radical cation
}

Cite as: J. Chem. Phys. 153, 031102 (2020); https://doi.org/10.1063/5.0015937

Submitted: 01 June 2020 . Accepted: 25 June 2020 . Published Online: 15 July 2020

Thierry Tran (D), Andrew J. Jenkins (D), Graham A. Worth (D), and Michael A. Robb (D)

\section{ARTICLES YOU MAY BE INTERESTED IN}

\section{Modern quantum chemistry with [Open]Molcas}

The Journal of Chemical Physics 152, 214117 (2020); https://doi.org/10.1063/5.0004835

TeraChem: Accelerating electronic structure and ab initio molecular dynamics with graphical processing units

The Journal of Chemical Physics 152, 224110 (2020); https://doi.org/10.1063/5.0007615

The Molpro quantum chemistry package

The Journal of Chemical Physics 152, 144107 (2020); https://doi.org/10.1063/5.0005081

\section{Lock-in Amplifiers up to $600 \mathrm{MHz}$}
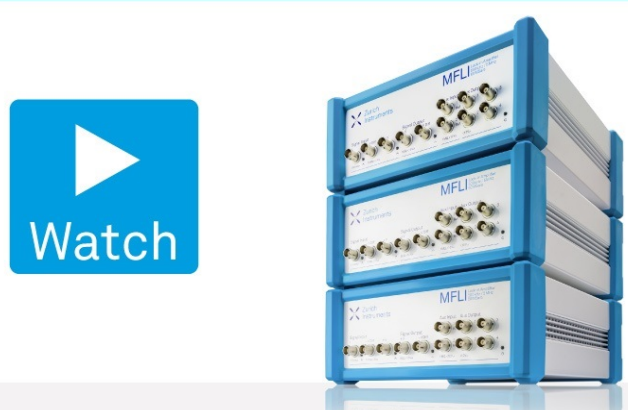

J. Chem. Phys. 153, 031102 (2020); https://doi.org/10.1063/5.0015937 


\title{
The quantum-Ehrenfest method
} with the inclusion of an IR pulse: Application to electron dynamics of the allene radical cation

\author{
Cite as: J. Chem. Phys. 153, 031102 (2020); doi: 10.1063/5.0015937 \\ Submitted: 1 June 2020 - Accepted: 25 June 2020 • \\ Published Online: 15 July 2020
}

\begin{abstract}
Thierry Tran, ${ }^{1, a)}$ (D) Andrew J. Jenkins, ${ }^{2, b)}$ (D) Graham A. Worth, ${ }^{1, c)}$ (D) and Michael A. Robb邽, (D)
AFFILIATIONS

'Department of Chemistry, University College London, 20, Gordon St., WC1H OAJ London, United Kingdom

2Department of Chemistry, University of Washington, Seattle, Washington 98195, USA

${ }^{3}$ Department of Chemistry, Molecular Sciences Research Hub, Imperial College London, White City Campus, 80 Wood Lane, W12 OBZ London, United Kingdom
\end{abstract}

a)thierry.tran.18@ucl.ac.uk
b) jenkins6@uw.edu
c) g.a.worth@ucl.ac.uk

d) Author to whom correspondence should be addressed: mike.robb@imperial.ac.uk

\begin{abstract}
We describe the implementation of a laser control pulse in the quantum-Ehrenfest method, a molecular quantum dynamics method that solves the time-dependent Schrödinger equation for both electrons and nuclei. The oscillating electric field-dipole interaction is incorporated directly in the one-electron Hamiltonian of the electronic structure part of the algorithm. We then use the coupled electron-nuclear dynamics of the $\pi$-system in the allene radical cation $\left(\bullet \mathrm{CH}_{2}=\mathrm{C}=\mathrm{CH}_{2}\right)^{+}$as a simple model of a pump-control experiment. We start (pump) with a two-state superposition of two cationic states. The resulting electron dynamics corresponds to the rapid oscillation of the unpaired electron between the two terminal methylenes. This electron dynamics is, in turn, coupled to the torsional motion of the terminal methylenes. There is a conical intersection at $90^{\circ}$ twist, where the electron dynamics collapses because the adiabatic states become degenerate. After passing the conical intersection, the electron dynamics revives. The IR pulse (control) in our simulations is timed to have its maximum at the conical intersection. Our simulations show that the effect of the (control) pulse is to change the electron dynamics at the conical intersection and, as a consequence, the concomitant nuclear dynamics, which is dominated by the change in the torsional angle.
\end{abstract}

Published under license by AIP Publishing. https://doi.org/10.1063/5.0015937

\section{INTRODUCTION}

Molecular quantum dynamics simulations ${ }^{1}$ are an essential tool to provide a molecular-level and electron-level understanding of the results observed in spectroscopy experiments, particularly where the initial pulse creates a superposition of adiabatic states. ${ }^{2,3}$ The oscillating electric field of the pulse interacts with the electronic wavefunction, which, in turn, will modify the dynamics of the nuclear wavefunction due to the coupling between the electronic and nuclear motion. The goal of this work is to simulate the effect of a control pulse to manipulate the dynamics of the molecular wavefunction near a conical intersection. Some examples of other different algorithms for studying such effects can be found in the literature. To cite just a few examples, the previous work has included the effect of an external field in dynamics near a conical intersection by using a diabatic dipole moment and transition dipole moment dot product with the electric field ${ }^{4-6}$ and by using the optimal control formalism.

In a recent work, we have used our Quantum-Ehrenfest $(\mathrm{Qu}-$ Eh $)^{8}$ method to follow the electron dynamics associated with a pulse that creates a superposition of adiabatic states. In this paper, we discuss incorporating a subsequent IR control pulse in the Qu-Eh simulations. The Qu-Eh method is a quantum molecular dynamics method related to the Direct Dynamics 
variational-Multiconfiguration Gaussian (DD-vMCG) ${ }^{9}$ for nuclear motion but using an Ehrenfest method to describe the electrons. ${ }^{10}$ A comparison of both methods for different organic molecules was done by Jenkins et al. ${ }^{8}$ Both the electrons and nuclei are propagated on-the-fly using a variational solution of the time-dependent Schrödinger equation. The molecular wavefunction $\Psi(R, r, t)$ in $\mathrm{Qu}-$ Eh, starting from an exact factorization, ${ }^{11-13}$ is a function of the degrees of freedom of the nuclei $R$, the electrons $r$, and time $t$ and is given as a product of a time-dependent electronic wavefunction $\psi$ and a nuclear wavefunction $\chi$ [see Eq. (1)]. The nuclear wavefunction $\chi$ is a function of time and nuclear coordinates $R$ and is described with a basis of Gaussian wavepackets (GWPs) [see Eq. (2)]. The explicit form of a GWP is given in Eq. S1 of the supplementary material. The electronic wavefunction $\psi$ is a function of time and electron coordinates $r$ with the nuclear coordinate $R$ as a parameter and is described with a linear combination of many-electron basis states $\psi_{s}$ with coefficient $c_{s}$ varying in time [see Eq. (3)]. A review of the $\mathrm{Qu}-\mathrm{Eh}$ algorithm is given in the supplementary material,

$$
\begin{gathered}
\Psi(R, r, t)=\chi(R, t) \psi(r, t ; R), \\
\chi(R, t)=\sum_{i} A_{i}(t) g_{i}(R, t), \\
\psi(r, t ; R)=\sum_{s} c_{s}(t) \psi_{s}(r ; R) .
\end{gathered}
$$

For allene, the Ehrenfest electronic structure method is used with a complete active space configuration interaction (CAS-CI) ${ }^{8,14}$ method with an active space of three electrons in two localized $\pi$ orbitals, as shown in Fig. 1. The nuclear dynamics is described within Qu-Eh using a set of 16 GWPs to represent the nuclear wavefunction, which evolves on the time-dependent Ehrenfest potential. ${ }^{11-13}$ The nuclear dynamics is propagated using the Qu-Eh method implemented in Quantics, ${ }^{15,16}$ which has been interfaced with a development version of Gaussian ${ }^{17}$ for the electronic structure method.

In Quantics, a set of vibrational normal mode (NM) coordinates is used as an orthogonal basis. The coordinates $Q$ employed for the dynamics correspond to a mass-frequency scaled normal coordinate displacement with respect to an initial reference geometry. ${ }^{18,19}$ The conversion between the Cartesian coordinate displacement (with respect to the reference geometry) $r_{\alpha}$ and the normal mode displacement $Q_{i}$ is given by Eq. (4). A matrix $K$ containing the normal modes $i$ expressed in mass-weighted coordinates $\alpha$, is required as input to transform the Cartesian coordinates into normal mode coordinates, which have been obtained from a frequency

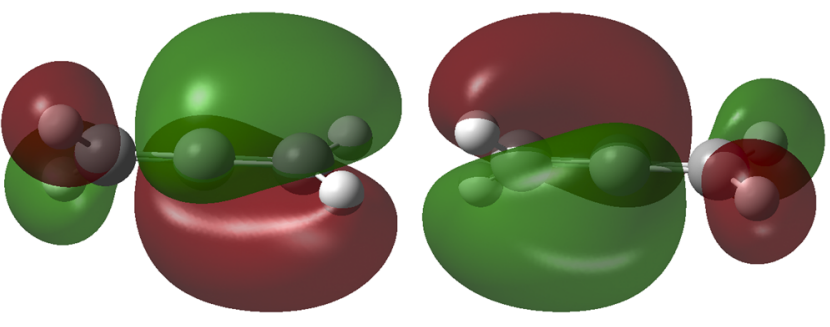

FIG. 1. Localized $\pi$ orbitals included in the active space. calculation. The coordinate $Q_{i}$ is then obtained by scaling it with the mass $M_{\alpha}$ of the atom of coordinate $\alpha$ and the frequency $\omega_{i}$ of normal mode $i$,

$$
Q_{i}=\sqrt{\frac{\omega_{i}}{\hbar}} \sum_{\alpha} K_{\alpha i} \sqrt{M_{\alpha}} r_{\alpha}
$$

The initial conditions correspond to (i) an initial superposition of the adiabatic electronic states and (ii) an initial nuclear wavepacket centered at the initial geometry, represented as a linear combination of 16 GWPs distributed in phase space with a momentum distribution, i.e., they have the same initial geometry but a different velocity for the center of the GWP. We also use a narrower width for the GWP to avoid numerical problems for the integration in the region of turning points (TPs) in electron dynamics (see the details of the initial conditions in the supplementary material).

The expectation values are evaluated by averaging over the 16 GWPs using the gross Gaussian population. ${ }^{20-22}$

\section{THEORY: INCLUDING THE EFFECT OF THE CONTROL PULSE IN QU-Eh DYNAMICS}

We now discuss including the effect of the control pulse in the $\mathrm{Qu}$-Eh dynamics algorithm. This is accomplished by adding a time-dependent term in the electronic Hamiltonian. ${ }^{23-25}$ The interaction between the electronic wavefunction and the oscillating electric field $\vec{E}(t)$ is included by adding the dipole-electric field term $\langle i|\vec{r}| j\rangle \cdot \vec{E}(t)$ to the one-electron electronic Hamiltonian $h_{e l}$, as shown in Eq. (5). The term $\left\langle i\left|h_{0}\right| j\right\rangle$ is just the nuclear attraction plus kinetic energy, and $\langle i|\vec{r}| j\rangle$ are the dipole integrals. This implementation is straightforward in the Qu-Eh method due to the use of a single time-dependent potential energy surface. Other methods of including an external electric field in dynamics have been accomplished by including the product of the field with the transition dipole moment in the equation of motion, ${ }^{26,27}$ propagator for semi-classical dynamics, ${ }^{28}$ a local-control method, ${ }^{29}$ and a Floquet Hamiltonian. ${ }^{30}$ A more detailed review can be found in the paper of Antipov et al, ${ }^{31}$

$$
h_{e l}(t)=\left\langle i\left|h_{0}\right| j\right\rangle+\langle i|\vec{r}| j\rangle \cdot \vec{E}(t) \text {. }
$$

The ionization process with the pump pulse is treated with the sudden approximation, where the dynamics is initiated on the superposition of cationic states. For the simulation, we just assume an initial electronic state that corresponds to the situation with a lone electron localized on one of the $\pi$ orbitals (Fig. 1). This situation corresponds to the superposition of the two adiabatic states. The initial geometry is the neutral ground state structure with the dihedral angle between the carbon and hydrogen atom set at $45^{\circ}$. The computational details are given in the supplementary material.

TABLE I. Parameters of the pulse included in dynamics given in SI units (left) and atomic units (right).

\begin{tabular}{lcc}
\hline \hline$E_{0}$ & $5 \times 10^{9} \mathrm{~V} / \mathrm{m}$ & 0.0098 a.u. \\
$\omega$ & $3.7 \times 10^{14} \mathrm{~Hz}$ & 0.05625 a.u. \\
$t_{0}$ & $15 \mathrm{fs}$ & 620.1206 a.u. \\
$\gamma$ & 0 & 0 \\
$w$ & $15 \mathrm{fs}^{-1}$ & 620.1206 a.u. \\
$\sigma$ & $0.0785 \mathrm{fs}^{-1}$ & 0.001899 a.u. \\
\hline
\end{tabular}


The pulse, as implemented in the Qu-Eh dynamics, models an experimental setup with a Ti:sapphire chirped-pulse amplification laser system with short IR pulses. ${ }^{32,33}$ The pulse $E(t)$ is represented [see Eqs. (6a) and (6b)] by a Gaussian centered at a $t_{0}$ of $15 \mathrm{fs}$ with a full-width at half maximum $w$ of $15 \mathrm{fs}$, a maximum electric field $E_{0}$ of $5 \times 10^{9} \mathrm{~V} / \mathrm{m}$, an oscillating electric field frequency $\omega$ of $3.7 \times 10^{14} \mathrm{~Hz}$ (the photon energy of $1.5307 \mathrm{eV}$ ), and a phase $\gamma$ of 0 (see Table I). The field is polarized in the direction of the molecular axis (along the $\mathrm{C}=\mathrm{C}$ bond), and a plot of the electric field profile can be found in Fig. $\mathrm{S} 1$ of the supplementary material,

$$
\begin{aligned}
E(t) & =E_{0} \cdot \cos \left(\omega\left(t-t_{0}\right)+\gamma\right) \cdot \exp \left(-\left(\sigma\left(t-t_{0}\right)\right)^{2}\right), \\
\sigma & =\sqrt{\frac{2 \ln (2)}{w^{2}}} .
\end{aligned}
$$

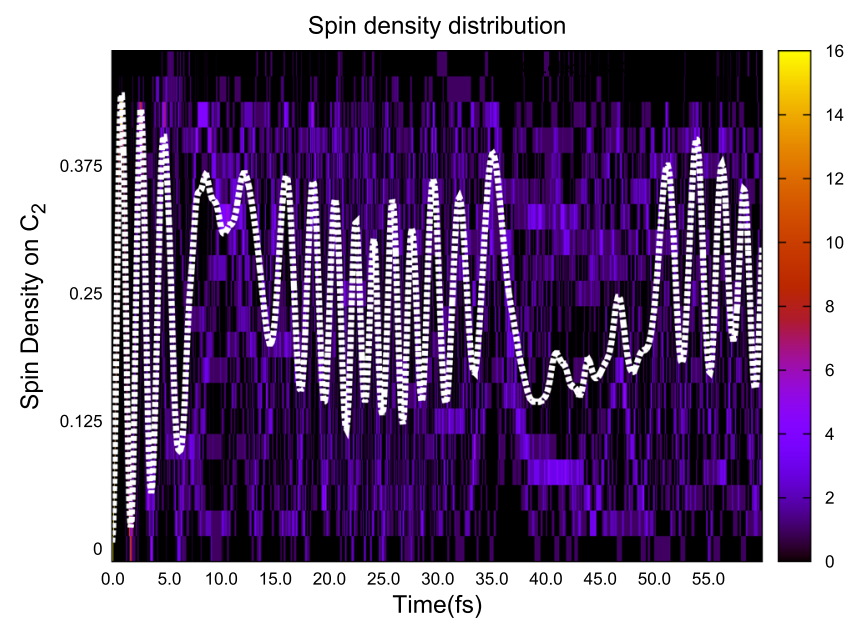

(a)

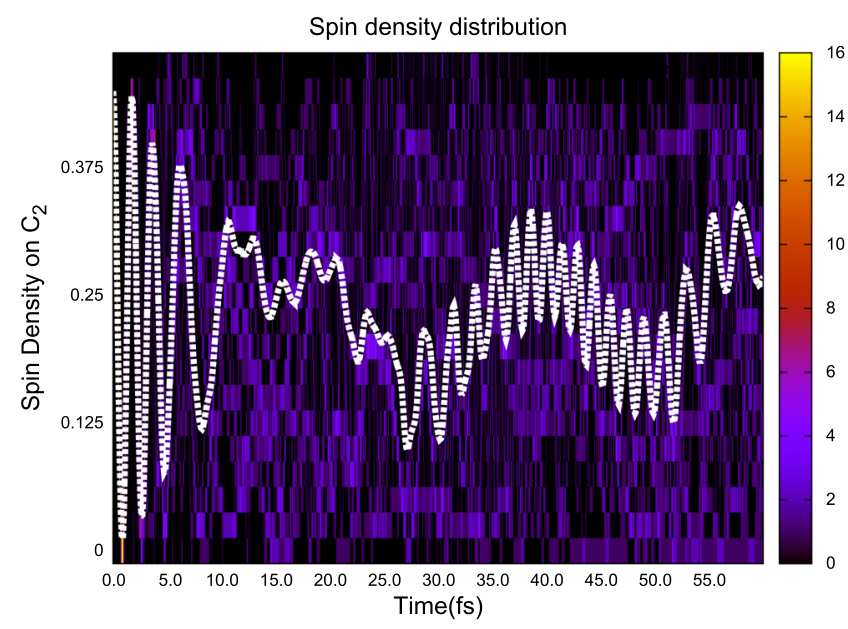

(b)

FIG. 2. Average spin density (in white) and its spread on the terminal carbon C2 for the 16 GWP dynamics (a) without a pulse and (b) with a pulse. Atom labeling is shown in Fig. S2 of the supplementary material.

\section{RESULTS AND DISCUSSION}

In the current work, we have included the interaction of an external electric field with the molecular wavefunction only at the electronic level directly in the one-electron Hamiltonian. However, further terms could be added; examples include the molecular dipole moment interaction into the equation of motion of the GWP or the derivative dipole moment in the calculation of the gradient of the potential. The pulse used in the dynamics as well as the orientation of the field with respect to the molecule has been chosen for investigating the non-resonant interaction ${ }^{34}$ of the field with electron dynamics with the purpose of steering the dynamics near a conical intersection.

The time-evolution of the system can be followed either by monitoring the evolution of the center of the nuclear wavefunction or by monitoring individual GWPs. We shall discuss the overall behavior of the complete nuclear wavepacket first using the spin

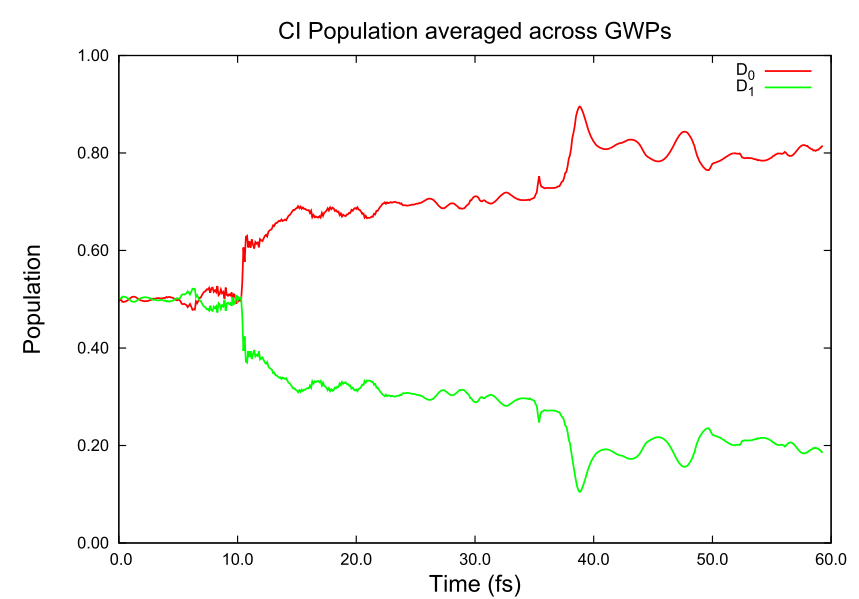

(a)

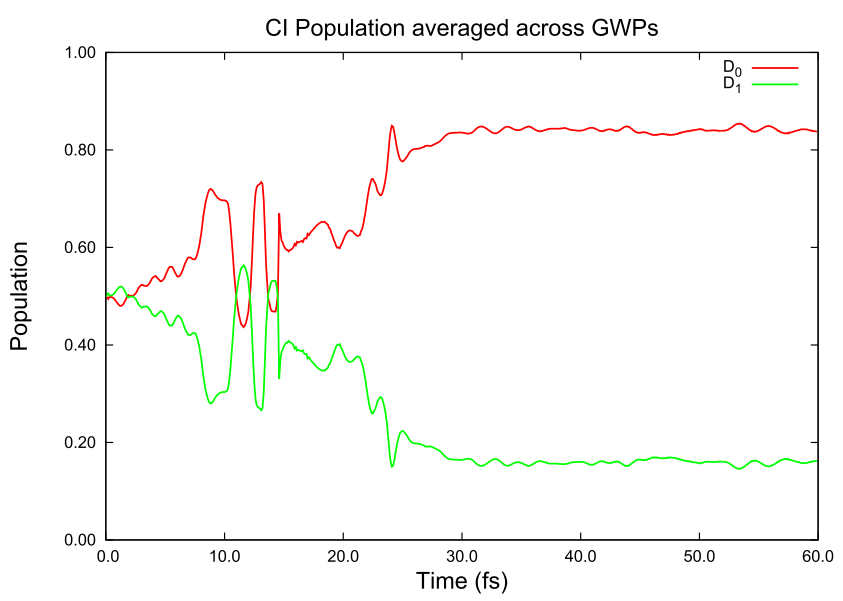

(b)

FIG. 3. Adiabatic state populations averaged over GWPs for (a) no pulse and (b) with pulse dynamics. 
density and then via normal mode displacement analysis. We focus our analysis on the torsional motion and the asymmetric $\mathrm{C}=\mathrm{C}$ bond stretching that spans the branching space for the lowest two cationic states of allene. ${ }^{35} \mathrm{We}$ also look at the symmetric $\mathrm{C}=\mathrm{C}$ stretching to monitor the overall change for the $\mathrm{C}=\mathrm{C}$ bond length due to the location of the charge. Subsequently, we will also look at the behavior, in detail, of one of the individual Gaussian wavepackets, GWP 6, that corresponds to an initial excitation of the normal mode for torsion. A similar analysis of all the 16 individual GWPs can be found in the supplementary material. The basis of 16 GWPs was employed to fill the half-shell in the momentum distribution (i.e., only the positive momentum of each normal mode is described with a function). In the current work, we do not focus on the convergence of the dynamics with the number of basis functions. However, it is known in the literature that full quantum dynamics methods can converge with a small number of basis functions, ${ }^{9,36}$ and the basis chosen resulted in functions that retain a low weight through the propagation (Sec. 7 of the supplementary material), which is an indication that it is large enough for reasonable results.

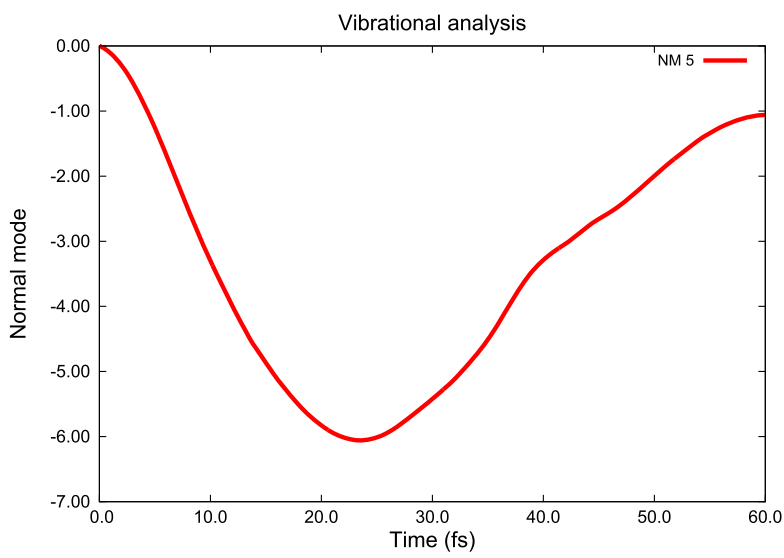

(a)

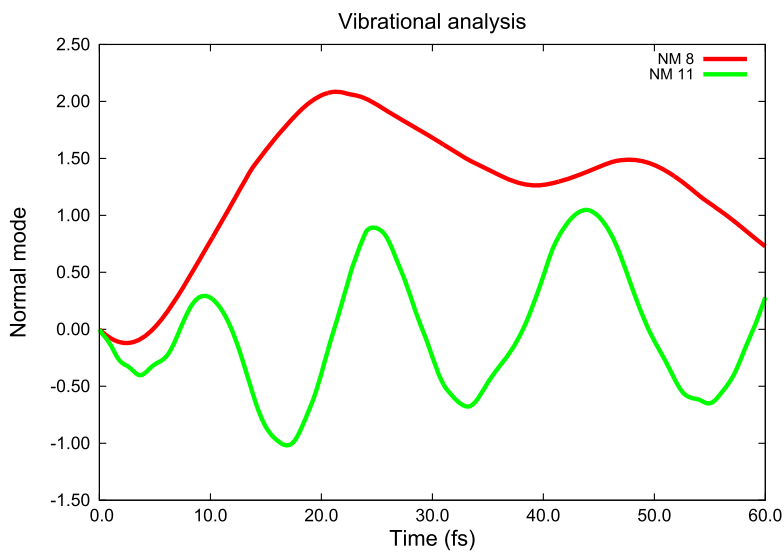

(c)
We have chosen to follow the spin density of the unpaired electron to understand the electron dynamics. The spin density provides us with information about the location of the lone electron and has the advantage that it is not dependent on the details of the electronic structure method. ${ }^{37}$ The analysis of the propagation of the nuclear wavefunction is done in the normal mode displacement basis (averaged over the weights of the individual GWPs; see Table S1 for the gross Gaussian population of the GWP at different times). We use this representation for the GWP because of the orthogonality of this representation and the ability to easily identify the relevant degrees of freedom compared to the Cartesian representation where the degrees of freedom are strongly coupled together.

We first discuss the electron dynamics, which we visualize with the spin density on the terminal carbon atom $2\left(\mathrm{C}_{2}\right)$ (averaged using the weights of the GWP). The complementary data for carbon atom $3\left(\mathrm{C}_{3}\right)$ can be found in Fig. S4 of the supplementary material. The labeling of the atom can be found in Fig. S2 of the supplementary material. In Fig. 2, we show the average spin density (in white) as well

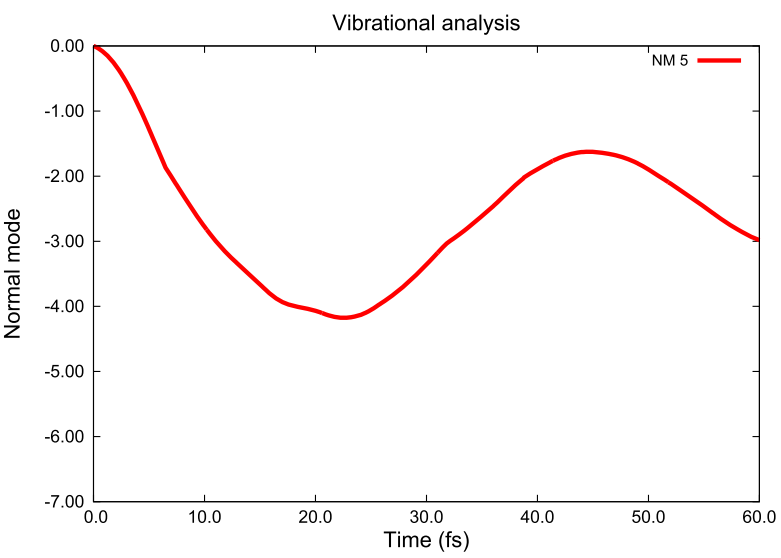

(b)

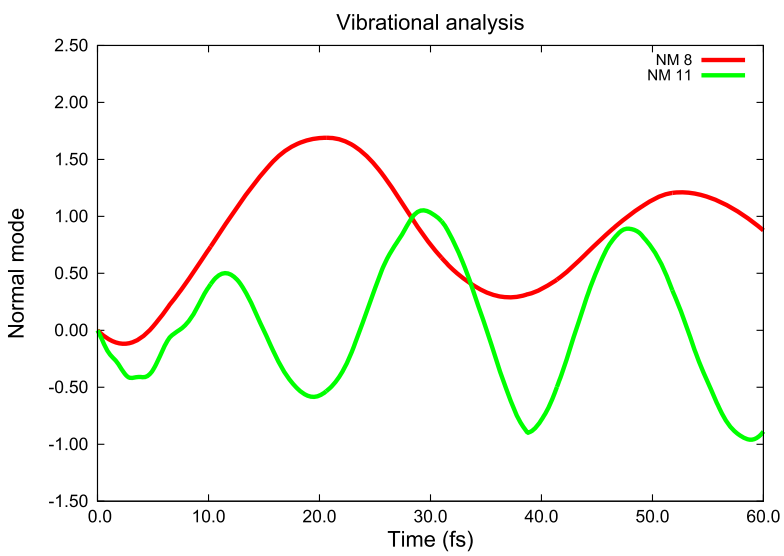

(d)

FIG. 4. Normal displacement for the center of the nuclear wavefunction. The torsional motion with normal mode 5: (a) without a pulse and (b) with a pulse. C-C stretching and $\mathrm{H}-\mathrm{C}-\mathrm{H}$ bending with normal modes 8 and 11: (c) without a pulse and (d) with a pulse. 
TABLE II. Turning point (TP)/torsional angle of NM 5 in Fig. 6.

\begin{tabular}{lll}
\hline \hline Time $(\mathrm{fs})$ & No pulse & Pulse \\
\hline 23 & $113(\mathrm{TP})$ & $95(\mathrm{TP})$ \\
48 & 73 & $67(\mathrm{TP})$ \\
60 & 58 & 81 \\
\hline \hline
\end{tabular}

as the spread in this distribution (corresponding to the contributions of the individual 16 GWPs, which are displayed using a blue color gradient). In Fig. 3, we show the corresponding averaged adiabatic populations.

In general, the period of the electron dynamics depends on the energy gap between the two states that corresponds to the location of an unpaired electron on either $\mathrm{C}_{2}$ or $\mathrm{C}_{3}$ : the larger the energy difference is, the shorter the period of the oscillations will be. Thus, at a conical intersection where the energy is zero, the period is infinite and the electron dynamics collapses. Thus, the change in the period of the electron dynamics reflects a change in the energy gap as the geometry is changed.

We now discuss the case where we have no pulse and where the dynamics begins with an equal superposition of the two adiabatic states. Looking at Fig. 2(a), we can see that there are four distinct phases of the electron dynamics: segment 1: $0 \mathrm{fs}-8 \mathrm{fs}$, where we have oscillatory electron dynamics and the unpaired electron moves between the two carbon atoms, segment 2: $8 \mathrm{fs}-15 \mathrm{fs}$, where the system passes near the conical intersection, segment 3: $15 \mathrm{fs}-35 \mathrm{fs}$, where the electron dynamics revives again, and segment 4: $35 \mathrm{fs}$ $-55 \mathrm{fs}$, where we again pass the conical intersection. In contrast, for the case where the pulse is switched on, Fig. 2(b), we see that segments 1 up to 8 fs are not changed very much. However, beginning in segment 2-3: $8 \mathrm{fs}-25 \mathrm{fs}$, we see that the electron dynamics only partially collapses and continues with a longer period (larger gap) until $25 \mathrm{fs}$. At $25 \mathrm{fs}$, the electron dynamics partly recovers and it is composed of a slow and fast oscillation.

Thus, the electron dynamics of the average of the 16 GWPs is strongly affected by the pulse particularly in the conical intersection region near 15 fs. However, a large spread can also be observed for the spin density on the individual GWP, as shown by the purple lines in Fig. 2. Thus, one must look at the individual GWP for a detailed understanding, and we return to this subsequently.

The response to the pulse also manifests itself in the adiabatic state populations shown in Fig. 3. On comparing segment 1: $0 \mathrm{fs}-8 \mathrm{fs}$ in Figs. 3(a) and 3(b), we can see that the pulse partly destroys the

TABLE III. Bond length in angstrom for $C_{1}-C_{2}$ and $C_{1}-C_{3}$ for the sum of NM 8 and NM 11 in Fig. 6.

\begin{tabular}{lcccc}
\hline \hline Time (fs) & No pulse & \multicolumn{3}{c}{ Pulse } \\
\hline & $\mathrm{C}_{1}-\mathrm{C}_{2}$ & $\mathrm{C}_{1}-\mathrm{C}_{3}$ & $\mathrm{C} 1-\mathrm{C} 2$ & $\mathrm{C}_{1}-\mathrm{C}_{3}$ \\
\hline 17 & 1.41 & 1.32 & 1.37 & 1.34 \\
40 & 1.33 & 1.37 & 1.36 & 1.29 \\
60 & 1.34 & 1.32 & 1.38 & 1.30 \\
\hline \hline
\end{tabular}

equal superposition of the two adiabatic states from the outset ( $0 \mathrm{fs}$ ). There is then a partial collapse and revival of the adiabatic populations in the conical intersection region (segment 2). It remains to try to understand this behavior of the adiabatic state populations and the spin density in terms of the nuclear motion.

Accordingly, we now turn to the motion of the nuclei in response to the electron dynamics. The motions of the nuclei are monitored either by following a given normal mode displacement coordinate $Q_{i}$ [Eq. (4)] for the center of the nuclear wavepacket or by using the nuclear displacement of a specific GWP $i+1$, where the initial conditions correspond to an initial momentum in that given normal mode $Q_{i}$. The changes in the period of the electron dynamics arise from the changes in the nuclear motion. Since the electron dynamics involves the carbon atoms of the two terminal methylenes, we expect that the important nuclear motion involves the torsion and the $\mathrm{C}-\mathrm{C}-\mathrm{C}$ stretches. These normal modes are shown in Fig. S3 of the supplementary material with arrows showing the direction of motion of the individual atom.

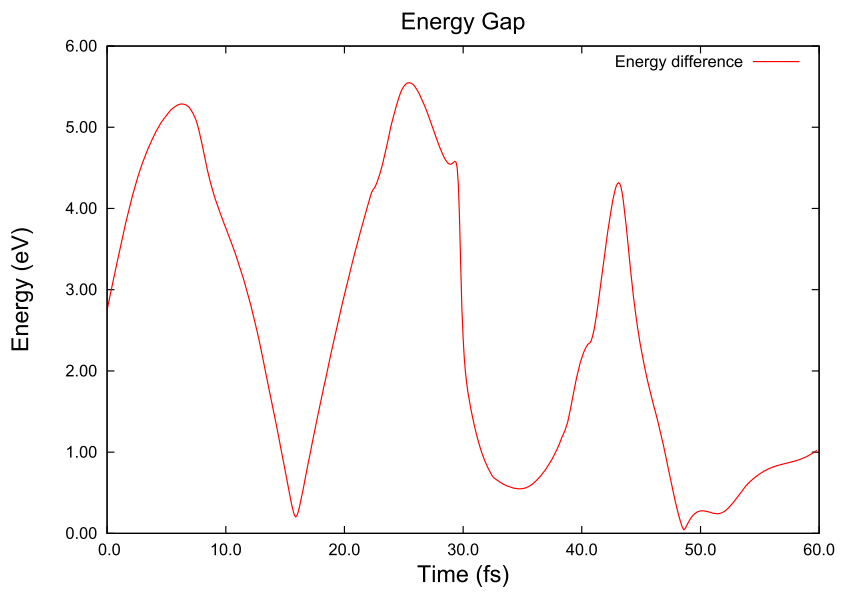

(a)

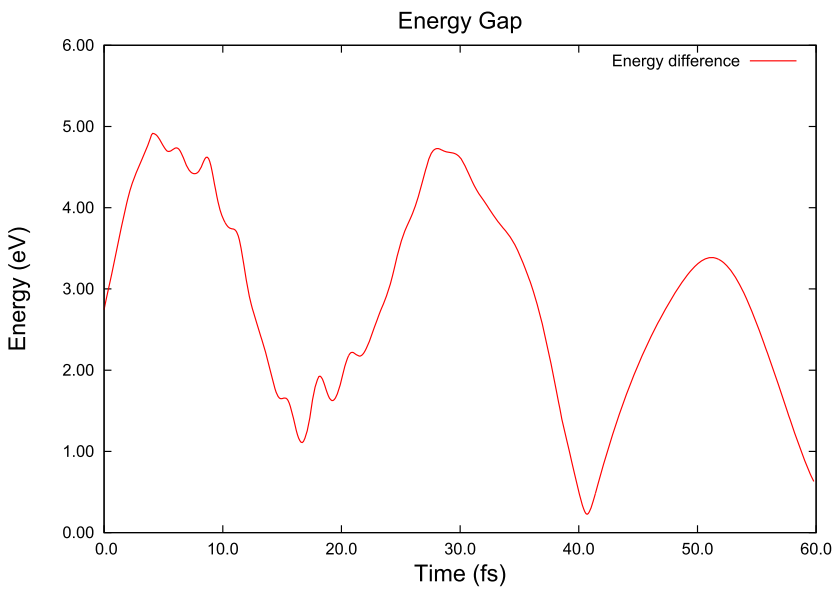

(b)

FIG. 5. Energy gap between the two adiabatic states as a function of time for GWP 6 dynamics (a) without a pulse and (b) with a pulse. 
In Figs. 4(a) and 4(b) and Table II, we collect the data for the torsional motion (normal mode 5). In Table II, we report the data for one specific dihedral angle $\left(\mathrm{H}_{4}-\mathrm{C}_{2}-\mathrm{C}_{3}-\mathrm{H}_{6}\right)$ to provide an alternative interpretation of Figs. 4(a) and 4(b), which effectively shows the combined motion of all four HCCH dihedral angles in the dimensionless units used for normal mode motion. The plot of the dihedral angle for the four $\mathrm{HCCH}$ where some points were used for Table II can be found in Fig. S5a of the supplementary material. Without the pulse, the center of the nuclear wavefunction follows the path from $45^{\circ}$ to $113^{\circ}$ (the turning point at $23 \mathrm{fs}$ ) and, thus, goes through the conical intersection $\left(90^{\circ}\right)$ two times, as shown in Fig. 6(a) (the normal mode shows a motion down to -6 units and back). With the presence of the pulse, the turning point still occurs at $23 \mathrm{fs}$ with a smaller angle $\left(95^{\circ}\right)$ (with a value of -4 units). With the pulse, there is a turning point at $67^{\circ}$ at $48 \mathrm{fs}$. While for the case of without the pulse, the torsional motion continues. Thus, without the pulse, there is a large amplitude torsion motion $45^{\circ} \rightarrow 90^{\circ} \rightarrow 113^{\circ} \rightarrow 90^{\circ} \rightarrow 73^{\circ}$ $\rightarrow 58^{\circ}$. In contrast, with the pulse, the torsion is very "tight": $45^{\circ} \rightarrow 90^{\circ} \rightarrow 95^{\circ}(\mathrm{TP}) \rightarrow 67^{\circ}(\mathrm{TP}) \rightarrow 81^{\circ}$ (i.e., between $95^{\circ}$ and $97^{\circ}$ ).
Now, we focus on the $\mathrm{C}-\mathrm{C}-\mathrm{C}$ stretching motion represented by the symmetric stretch with normal mode 8 and the asymmetric stretch with normal mode 11 [see Figs. 4(c) and 4(d) and Table III]. The atomic displacements corresponding to motion in these modes are shown in Figs. S3b and S3c of the supplementary material and Table III. Without the pulse, normal mode 8 shows a motion up to a value of 2 units with normal mode 11 reaching a minimum of -1 units, which corresponds roughly to a bond length of $1.41 \AA$ for $\mathrm{C}_{1}-\mathrm{C}_{2}$ and $1.32 \AA$ for $\mathrm{C}_{1}-\mathrm{C}_{3}$. Both $\mathrm{C}=\mathrm{C}$ bond lengths show little variation, with the change oscillating between 1.3 and $1.4 \AA$. With the presence of a pulse, smaller bond stretching is observed for the bond length which is shown by a smaller amplitude for NM 8.

It now remains to understand the relationship of the nuclear motion (Fig. 4 and Tables II and III) and the electron dynamics (shown in Fig. 2) focusing on the energy gap between the two electronic states. Let us look at the energy gap for the GWP corresponding to normal mode 5 (GWP 6) shown in Fig. 5. The energy gap plot for dynamics initiated in the direction of normal modes

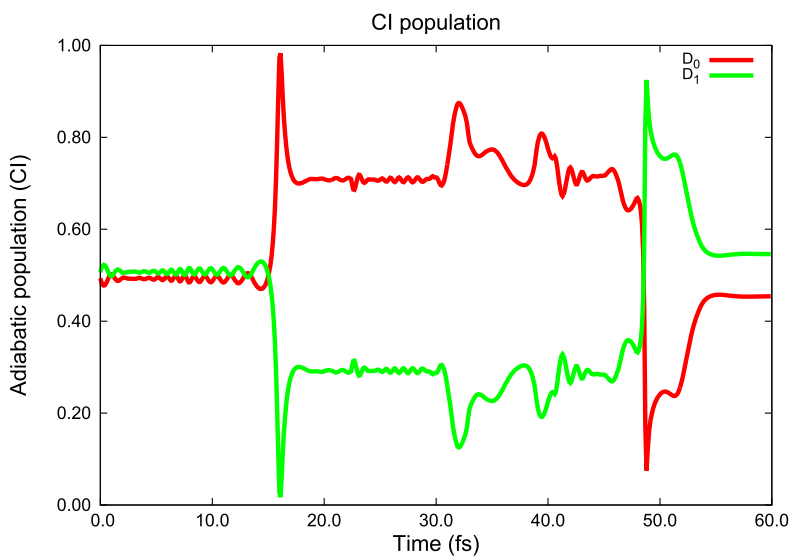

(a)

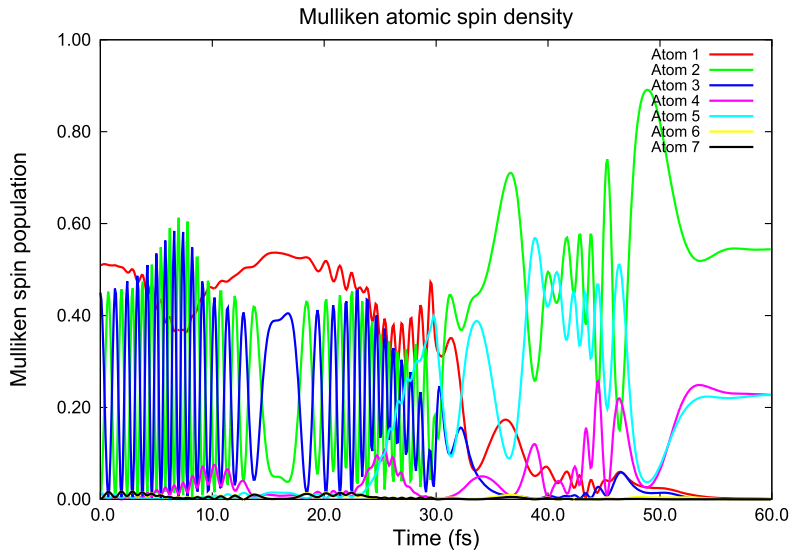

(c)

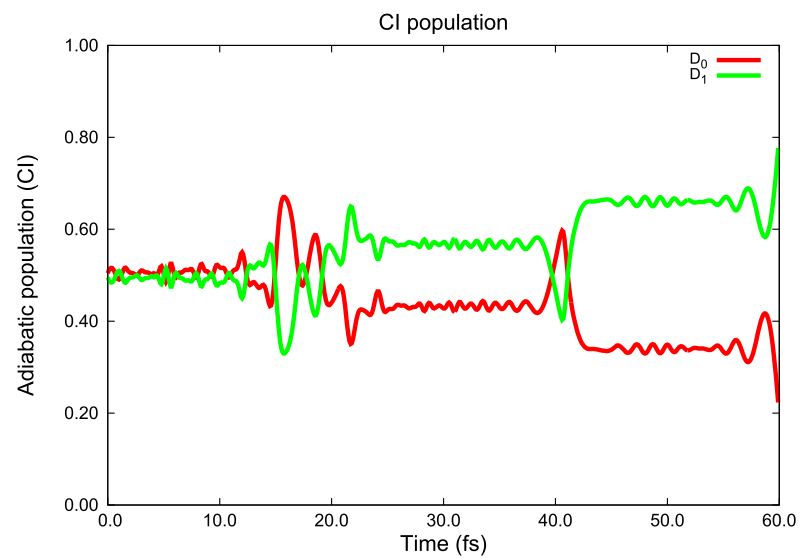

(b)

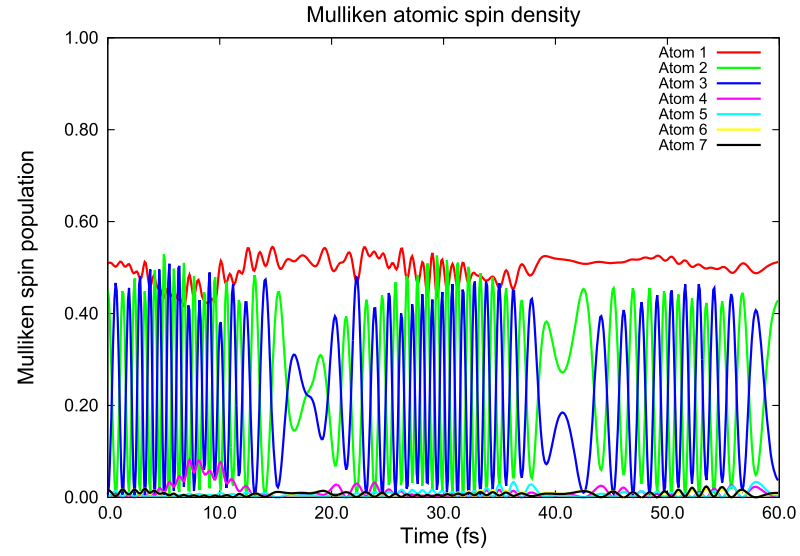

(d)

FIG. 6. Adiabatic populations along GWP 6: (a) no pulse adiabatic state populations, (b) adiabatic state populations with a pulse, and [(c) and (d)] their corresponding spin densities. 
8 and 11 with GWPs 9 and 12 can be found in Fig. S6 of the supplementary material. We can see that the behavior for the stretch modes (see Fig. $\mathbf{S 6}$ of the supplementary material) is hardly affected by the pulse. In contrast, in Figs. 5(a) and 5(b), we can see that the behavior of the energy gap before and after the pulse is very different. Thus, it would appear that the electron dynamics and the torsional motion are strongly coupled, but the stretching is hardly affected by the pulse. For example, at 15 fs without a pulse [Fig. 5(a)], the energy gap is almost zero, in correspondence with the collapse of the electron dynamics between $5 \mathrm{fs}$ and $15 \mathrm{fs}$ in Figs. 2(a) and 2(b). In contrast, in Fig. 5(b), we see a substantial gap at 15 fs. Thus, between $5 \mathrm{fs}$ and $15 \mathrm{fs}$, in the case of the presence of the pulse, we see electron dynamics with a longer period (i.e., small but a non-zero gap).

The preceding discussion has been focusing mainly on the normal mode motion averaged over the whole nuclear wavefunction. Furthermore, the adiabatic population shown in Fig. 3 is for the complete nuclear wavefunction. In contrast, if we look at individual GWPs (isolated points from the complete nuclear wavefunction) as in Fig. 5, we can obtain additional physical insight. The individual GWPs represent points that were initiated with velocity on a particular normal mode in the momentum distribution. Thus, GWP 6 corresponds to the GWP that was started with normal mode 5 being excited. The results for all GWPs can be found in the supplementary material: Fig. S7 for the adiabatic population, Fig. S8 for the spin density, and Fig. S9 for the dihedral angle. Comparing with Fig. 5, Fig. 6 shows the adiabatic populations along GWP 6. Note that GWP 6 decays to the ground state at $15 \mathrm{fs}$. In contrast, the pulse maintains the mixed state that leads to electron dynamics, which manifests itself in the change in spin density. Thus, in Figs. 6(a) and 6(c), without the pulse, the electron dynamics collapses at $15 \mathrm{fs}$. In contrast, with the pulse, the electron dynamics persists. The effect of the pulse is shown more emphatically by examining the spin density of the individual atoms [see Figs. 6(c) and 6(d)]. Without the pulse, there is an oscillation of the spin density between the two terminal carbon atoms for $30 \mathrm{fs}$ before the lone electron becomes localized onto one specific carbon atom (atom 2), whereas in the presence of the pulse, the charge migration persists beyond $30 \mathrm{fs}$ with a spin density oscillation between the two terminal carbon atoms (atom 2 and atom 3).

\section{CONCLUSIONS}

In this work, our objective has been to describe the effect, on nuclear-electron dynamics, of including the field of a short probe IR pulse in the electronic structure method. The pulse was applied near the conical intersection geometry. We have used a two-level electronic state model for the allene cation so that the results can be easily understood. The electron dynamics was observed by monitoring the spin density on the carbon atoms of the two methylene groups. The pulse strongly perturbs the electron dynamics at the conical intersection. As a consequence, the nuclear motion is changed and we observe a smaller displacement amplitude for both the torsional motion and the $\mathrm{C}-\mathrm{C}$ stretching with the presence of the pulse. The individual GWPs have a wide range of behaviors for the electron and nuclear motion. The average allows us to get a global picture on the effect of the pulse on the dynamics of the complete nuclear wavefunction.
The possibility of a direct study on how a short pulse affects the molecular wavefunction opens the door for investigating how fast electron dynamics interacting with an electric field can influence the nuclear dynamics.

\section{SUPPLEMENTARY MATERIAL}

See the supplementary material for computational details, initial conditions, the $\mathrm{Qu}-\mathrm{Eh}$ equation and algorithm, the form of the pulse, normal modes, averaged results for the spin density and torsional parameter, and results for individual wavepackets.

\section{ACKNOWLEDGMENTS}

The authors are grateful for financial support from Gaussian, Inc. and the UK Engineering and Physical Science Council (Grant No. EP/T006943/1). We also acknowledge discussions with H. B. Schlegel (Wayne State University) and X. Li (University of Washington).

\section{DATA AVAILABILITY}

The data that support the findings of this study are available within the article and its supplementary material. All computations were carried out at the Imperial College Research Computing Service (DOI: 10.14469/hpc/2232).

\section{REFERENCES}

${ }^{1}$ B. F. E. Curchod and T. J. Martínez, Chem. Rev. 118, 3305 (2018).

${ }^{2}$ G. Sansone, F. Kelkensberg, J. F. Pérez-Torres, F. Morales, M. F. Kling, W. Siu, O. Ghafur, P. Johnsson, M. Swoboda, E. Benedetti, F. Ferrari, F. Lépine, J. L. Sanz-Vicario, S. Zherebtsov, I. Znakovskaya, A. L'Huillier, M. Y. Ivanov, M. Nisoli, F. Martín, and M. J. J. Vrakking, Nature 465, 763 (2010).

${ }^{3}$ D. Mendive-Tapia, M. Vacher, M. J. Bearpark, and M. A. Robb, J. Chem. Phys. 139, 044110 (2013).

${ }^{4}$ Y. Arasaki and K. Takatsuka, Phys. Chem. Chem. Phys. 12, 1239 (2010).

${ }^{5}$ T. Rozgonyi and L. González, Chem. Phys. Lett. 459, 39 (2008).

${ }^{6}$ B. J. Rao, M. F. Gelin, and W. Domcke, J. Chem. Phys. 146, 084105 (2017).

${ }^{7}$ K. R. Nandipati, A. K. Kanakati, H. Singh, Z. Lan, and S. Mahapatra, Eur. Phys. J. D 71, 222 (2017).

${ }^{8}$ A. J. Jenkins, K. E. Spinlove, M. Vacher, G. A. Worth, and M. A. Robb, J. Chem. Phys. 149, 094108 (2018).

${ }^{9}$ G. W. Richings, I. Polyak, K. E. Spinlove, G. A. Worth, I. Burghardt, and B. Lasorne, Int. Rev. Phys. Chem. 34, 269 (2015).

${ }^{10}$ M. Vacher, M. J. Bearpark, and M. A. Robb, Theor. Chem. Acc. 135, 187 (2016).

${ }^{11}$ F. Agostini, S. K. Min, A. Abedi, and E. K. U. Gross, J. Chem. Theory Comput. 12, 2127 (2016).

${ }^{12}$ A. Abedi, N. T. Maitra, and E. K. U. Gross, J. Chem. Phys. 137, 22A530 (2012).

${ }^{13}$ A. Abedi, N. T. Maitra, and E. K. U. Gross, Phys. Rev. Lett. 105, 123002 (2010).

${ }^{14}$ A. J. Jenkins and M. A. Robb, Comput. Theor. Chem. 1152, 53 (2019).

${ }^{15}$ G. A. Worth, K. Giri, G. W. Richings, I. Burghardt, M. H. Beck, A. Jäckle, and H. D. Meyer, The QUANTICS Package, 2015.

${ }^{16}$ G. A. Worth, Comput. Phys. Commun. 248, 107040 (2020).

${ }^{17}$ M. J. Frisch, G. W. Trucks, H. B. Schlegel, G. E. Scuseria, M. A. Robb, J. R. Cheeseman, G. Scalmani, V. Barone, G. A. Petersson, H. Nakatsuji, X. Li, M. Caricato, A. V. Marenich, J. Bloino, B. G. Janesko, R. Gomperts, B. Mennucci, H. P. Hratchian, J. V. Ortiz, A. F. Izmaylov, J. L. Sonnenberg, D. Williams-Young, F. Ding, F. Lipparini, F. Egidi, J. Goings, B. Peng, A. Petrone, T. Henderson, D. Ranasinghe, V. G. Zakrzewski, J. Gao, N. Rega, G. Zheng,W. Liang, M. Hada, 
M. Ehara, K. Toyota, R. Fukuda, J. Hasegawa, M. Ishida, T. Nakajima, Y. Honda, O. Kitao, H. Nakai, T. Vreven, K. Throssell, J. A. Montgomery, Jr., J. E. Peralta, F. Ogliaro, M. J. Bearpark, J. J. Heyd, E. N. Brothers, K. N. Kudin, V. N. Staroverov, T. A. Keith, R. Kobayashi, J. Normand, K. Raghavachari, A. P. Rendell, J. C. Burant, S. S. Iyengar, J. Tomasi, M. Cossi, J. M. Millam, M. Klene, C. Adamo, R. Cammi, J. W. Ochterski, R. L. Martin, K. Morokuma, O. Farkas, J. B. Foresman, and D. J. Fox, Gaussian Development Version J.02, Gaussian, Inc., Wallingford, CT, 2018.

${ }^{18}$ F. Plasser, S. Gómez, M. F. S. J. Menger, S. Mai, and L. González, Phys. Chem. Chem. Phys. 21, 57 (2019).

${ }^{19}$ G. A. Worth and L. S. Cederbaum, Annu. Rev. Phys. Chem. 55, 127 (2004).

${ }^{20}$ J. D. Coe, M. T. Ong, B. G. Levine, and T. J. Martińez, J. Phys. Chem. A 112, 12559 (2008).

${ }^{21}$ C. S. M. Allan, B. Lasorne, G. A. Worth, and M. A. Robb, J. Phys. Chem. A 114, 8713 (2010).

${ }^{22}$ D. Mendive-Tapia, B. Lasorne, G. A. Worth, M. A. Robb, and M. J. Bearpark, J. Chem. Phys. 137, 22A548 (2012)

${ }^{23}$ X. Li, S. M. Smith, A. N. Markevitch, D. A. Romanov, R. J. Levis, and H. B. Schlegel, Phys. Chem. Chem. Phys. 7, 233 (2005).

${ }^{24}$ K. Harumiya, H. Kono, Y. Fujimura, I. Kawata, and A. D. Bandrauk, Phys. Rev. A 66, 043403 (2002).

${ }^{25}$ M. Lein, T. Kreibich, E. K. U. Gross, and V. Engel, Phys. Rev. A 65, 033403 (2002).

${ }^{26}$ B. F. E. Curchod, T. J. Penfold, U. Rothlisberger, and I. Tavernelli, Chimia 67, 218 (2013).
${ }^{27}$ T. J. Penfold, M. Pápai, K. B. Møller, and G. A. Worth, Comput. Theor. Chem. 1160, 24 (2019)

${ }^{28}$ M. Y. Ivanov, O. V. Tikhonova, and M. V. Fedorov, Phys. Rev. A 58, 793 (1998).

${ }^{29}$ P. Marquetand, S. Gräfe, D. Scheidel, and V. Engel, J. Chem. Phys. 124, 054325 (2006).

${ }^{30}$ D. v. Makhov and D. v. Shalashilin, Chem. Phys. 515, 46 (2018).

${ }^{31}$ S. v. Antipov, S. Bhattacharyya, K. el Hage, Z.-H. Xu, M. Meuwly, U. Rothlisberger, and J. Vaníček, Struct. Dyn. 4, 061509 (2017).

${ }^{32}$ A. Ludwig, E. Liberatore, J. Herrmann, L. Kasmi, P. López-Tarifa, L. Gallmann, U. Rothlisberger, U. Keller, and M. Lucchini, J. Phys. Chem. Lett. 7, 1901 (2016).

${ }^{33}$ R. Locher, M. Lucchini, J. Herrmann, M. Sabbar, M. Weger, A. Ludwig, L. Castiglioni, M. Greif, M. Hengsberger, L. Gallmann, and U. Keller, Rev. Sci. Instrum. 85, 013113 (2014).

${ }^{34}$ B. J. Sussman, D. Townsend, M. Y. Ivanov, and A. Stolow, Science 314, 278 (2006).

${ }^{35}$ A. Markmann, G. A. Worth, and L. S. Cederbaum, J. Chem. Phys. 122, 144320 (2005).

${ }^{36}$ D. v. Shalashilin, Faraday Discuss. 153, 105 (2011).

${ }^{37}$ I. Polyak, M. J. Bearpark, and M. A. Robb, Int. J. Quantum Chem. 118, e25559 (2018).

${ }^{38}$ S. Gómez, M. Heindl, A. Szabadi, and L. González, J. Phys. Chem. A 123, 8321 (2019). 